\title{
Разрывы валентной зоны в напряженных слоях SiGeSn/Si с различным содержанием олова
}

\author{
(C) А.А. Блошкин ${ }^{1,2}$, А.И. Якимов ${ }^{1,3}$, В.А. Тимофееев ${ }^{1}$, А.Р. Туктамышев $^{1}$, \\ А.И. Никифоров ${ }^{1,3}$, В.В. Мурашов ${ }^{4}$ \\ ${ }^{1}$ Институт фризики полупроводников им. А.В. Ржанова \\ Сибирского отделения Российской академии наук, \\ 630090 Новосибирск, Россия \\ ${ }^{2}$ Новосибирский государственный университет, \\ 630090 Новосибирск, Россия \\ ${ }^{3}$ Томский государственный университет, \\ 634050 Томск, Россия \\ ${ }^{4}$ Новосибирский государственный технический университет, \\ 630073 Новосибирск, Россия \\ E-mail: bloshkin@isp.nsc.ru
}

(Получена 2 июня 2016 г. Принята к печати 14 июня 2016 г.)

Методом спектроскопии адмиттанса исследованы состояния дырок в квантовых ямах $\mathrm{Si}_{0.7-y} \mathrm{Ge}_{0.3} \mathrm{Sn}_{y} / \mathrm{Si}_{\text {в }}$ диапазоне содержания олова $y=0.04-0.1$. Установлено, что энергия связи дырки растет с увеличением содержания олова. В рамках 6-зонного kр-метода определены энергии размерного квантования дырок в структурах, содержащих псевдоморфный слой $\mathrm{Si}_{0.7-y} \mathrm{Ge}_{0.3} \mathrm{Sn}_{y}$ в матрице $\mathrm{Si}$. Комбинацией результатов математического моделирования и экспериментальных данных определена величина разрыва валентной зоны на гетерогранице $\mathrm{Si} / \mathrm{Si}_{0.7-y} \mathrm{Ge}_{0.3} \mathrm{Sn}_{y}$. Установлено, что зависимость экспериментальных значений разрыва валентных зон между псевдоморфными слоями $\mathrm{Si}_{0.7-y} \mathrm{Ge}_{0.3} \mathrm{Sn}_{y}$ и $\mathrm{Si}$ от содержания олова описывается выражением $\Delta E_{V}^{\exp }=(0.21 \pm 0.01)+\left(3.35 \pm 7.8 \cdot 10^{-4}\right)$ у эВ.

DOI: $10.21883 /$ FTP.2017.03.44205.8343

\section{1. Введение}

Кремний является основным материалом микро- и наноэлектроники, но в оптоэлектронике его применение ограниченно. Связано это с двумя особенностями материала. Во-первых, главный минимум зоны проводимости $\mathrm{Si}$ смещен относительно центра зоны Бриллюэна, что резко уменьшает эффективность излучающих структур на базе $\mathrm{Si}$. Во-вторых, ширина запрещенной зоны $\mathrm{Si}$ составляет 1.12 эВ при комнатной температуре, делая кремний прозрачным в среднем и дальнем инфракрасном (ИК) диапазонах. Из-за этого факта реализация фотонных ИК фотоприемников на базе чистого кремния вызывает проблемы. На преодоление указанных ограничений тратится много усилий, и применяются различные подходы, такие как использование сплавов элементов IV группы таблицы Менделеева и наноструктур на их основе. В разные годы предлагалось рассматривать гетероструктуры $\mathrm{GeSi}$ с квантовыми точками (КТ) [1-4]. Данный подход дал определенные результаты, но из-за малой плотности квантовых точек не удается получить значительную величину квантового выхода в фотоприемных устройствах. В качестве альтернативного пути рассматривается использование тройного соединения $\mathrm{SiGeSn}$ на подложках $\mathrm{Si}$. Для сплава $\mathrm{GeSn}$ предсказывалась теоретически [5,6] и наблюдалась экспериментально [7-9] смена характера фундаментального поглощения с непрямозонного на прямозонный при малых содержаниях олова. Полученные результаты дают надежду на реализацию светоизлучающих приборов на кремниевой подложке с тройным соединением $\mathrm{SiGeSn}$ в качестве активного элемента. Кроме того, поскольку $\alpha$-Sn является полупроводником с инвертированной зонной структурой, вариация содержания олова в сплавах дает значительно больший диапазон ширин запрещенной зоны, которые могут быть использованы в приборах на основе твердых растворов $\mathrm{SiGeSn}$, по сравнению с приборами на $\mathrm{SiGe}$. На данный момент имеется множество работ экспериментального характеpa, посвященных формированию тройных соединений $\mathrm{SiGeSn} \mathrm{[10-13],} \mathrm{измерению} \mathrm{оптических} \mathrm{свойств} \mathrm{этих}$ полупроводников [13-17] и изготовлению отдельных приборных структур с использованием рассматриваемых твердых растворов [18-20]. Существуют теоретические работы, посвященные моделированию зонной структуры как напряженных, так и релаксированных $[5,6]$ тройных соединений. Однако в большинстве таких работ рассматривается лишь вопрос о ширине запрещенной зоны в гетероструктурах и совсем не затрагивается проблема установления разрыва зон на гетерогранице $\mathrm{Si} / \mathrm{SiGeSn}$. Иногда разрыв валентных зон Ge и $\mathrm{Sn}$ рассчитывается в модели, предложенной в [21]. Вместе с тем величина разрыва зон на границе $\mathrm{Si} / \mathrm{SiGeSn}$ имеет важное значение для формирования приборных структур. В настоящей работе методом спектроскопии адмиттанса были исследованы гетероструктуры с квантовыми ямами (КЯ) $\mathrm{SiGeSn}$, сформированными в матрице $\mathrm{Si}$, и определены энергии локализации дырок и разрыв валентной зоны. 


\section{2. Методика эксперимента и обработки экспериментальных данных}

Образцы для измерения были изготовлены методом молекулярно-лучевой эпитаксии на подложках $p^{+}-\mathrm{Si}(001)$ (рис. 1,a). На поверхности предварительно очищенной подложки осаждался буферный слой кремния толщиной 0.5 мкм, легированный бором до концентрации $5 \cdot 10^{16} \mathrm{~cm}^{-3}$. Температура роста кремниевого слоя составляла $500^{\circ} \mathrm{C}$. Затем подложка охлаждалась до $100^{\circ} \mathrm{C}$ и выращивалась пленка твердого раствора $\mathrm{SiGeSn}$ толщиной 2 нм. Содержание олова $y$ варьировалось в различных образцах от 4 до $10 \%$ и определялось по соотношению потоков кремния, германия и олова. После формирования квантовой ямы вся структура закрывалась кремнием толщиной 150 нм с концентрацией

\begin{tabular}{|c|}
\hline $\mathrm{Al}$ \\
\hline$p-\mathrm{Si}: 500 \mathrm{~nm}, 500^{\circ} \mathrm{C}, 5 \cdot 10^{16} \mathrm{~cm}^{-3}$ \\
\hline $\mathrm{Si}_{0.7-y} \mathrm{Ge}_{0.3} \mathrm{Sn}_{y}: 100^{\circ} \mathrm{C}, 2 \mathrm{~nm}$ \\
\hline$p-\mathrm{Si}: 500 \mathrm{~nm}, 500^{\circ} \mathrm{C}, 5 \cdot 10^{16} \mathrm{~cm}^{-3}$ \\
\hline$p^{+}-\mathrm{Si}(100)$ \\
\hline
\end{tabular}

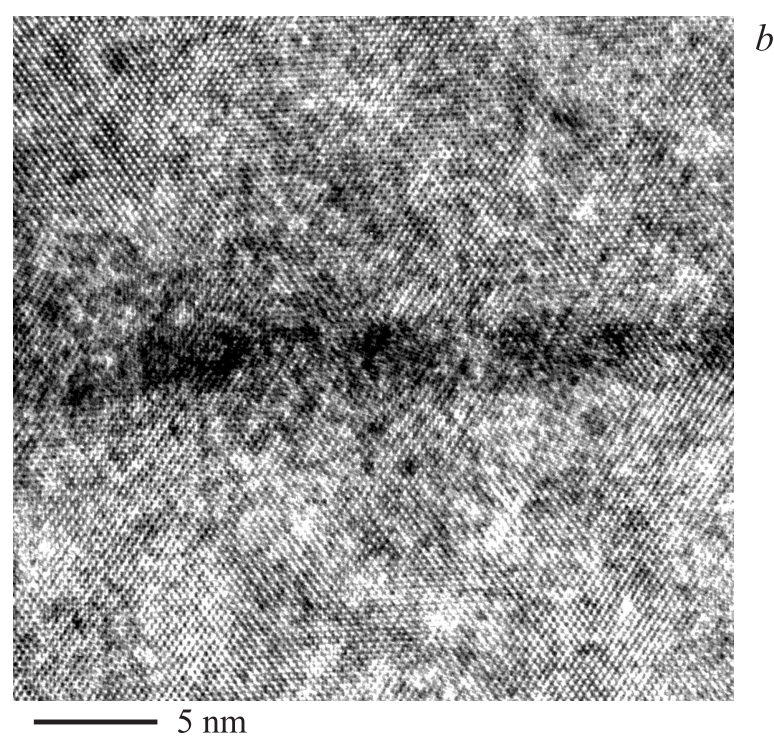

Рис. 1. Схематическое изображение гетероструктуры $\mathrm{Si} / \mathrm{SiGeSn} / \mathrm{Si}$, использовавшейся для измерений методом спектроскопии адмиттанса $(a)$, и изображение поперечного среза гетероструктуры, полученное с помощью просвечивающей электронной микроскопии $(b)$.

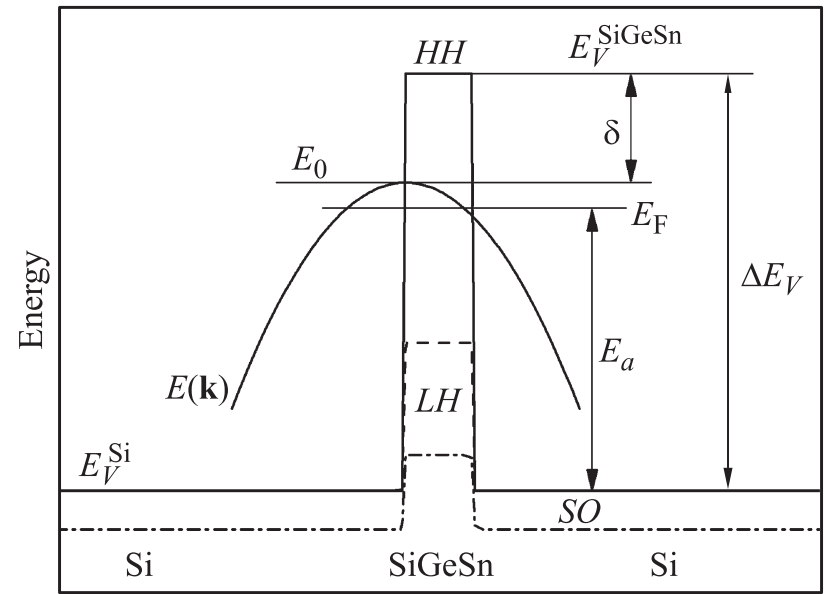

Distance in [001] direction

Рис. 2. Изображение профиля валентной зоны гетероструктуры $\mathrm{Si} / \mathrm{SiGeSn} / \mathrm{Si}$ вдоль направления роста. $H H, H L, S O-$ подзоны тяжелых, легких дырок и подзона, отщепленная спинорбитальным взаимодействием. Также показаны положение основного состояния дырки $E_{0}$ в КЯ и закон дисперсии $E(\mathbf{k})$.

бора $\sim 5 \cdot 10^{16} \mathrm{~cm}^{-3}$ при температуре $500^{\circ} \mathrm{C}$. В процессе роста верхнего слоя одновременно отжигались дефекты, возникающие в низкотемпературном слое $\mathrm{SiGeSn}$. Формирование барьеров Шоттки завершалось напылением в вакууме пленки Al. Содержание Ge во всех квантовых ямах составляло $30 \%$, что определялось следующими обстоятельствами. Во-первых, при данном составе твердого раствора на подложках $\mathrm{Si}$ можно вырастить достаточно толстые псевдоморфные слои $\mathrm{SiGe}$ без перехода от двумерного к трехмерному росту [22]. Во-вторых, при таком составе возможно формирование твердого раствора с содержанием олова до $10 \%$, потому что рассогласование параметров решетки твердый раствор-подложка не превышает 3\%. Изображение поперечного среза структуры, полученное с помощью просвечивающей электронной микроскопии (рис. 1,b), показывает высокое кристаллическое качество гетероструктуры.

На рис. 2 приведена схематическая зонная диаграмма гетероструктуры $\mathrm{Si} / \mathrm{SiGeSn} / \mathrm{Si}$, показано положение основного состояния дырки и закон дисперсии. Для определения энергии связи дырки в КЯ использовался метод спектроскопии адмиттанса, описанный ранее в работах [23-26]. Суть метода состоит в измерении проводимости системы на переменном токе, возникающей при изменении концентрации дырок в слое SiGeSn вследствие эмиссии носителей заряда из пленки в матрицу $\mathrm{Si}$ и их обратного захвата. При термически стимулированной эмиссии дырок через барьер в гетероструктуре с КЯ эффективная параллельная проводимость достигает максимума при температуре $T_{\max }$, удовлетворяющей условию

$$
f=\alpha T_{\max } \exp \left(-E_{a} / k_{\mathrm{B}} T_{\max }\right),
$$

где $\alpha-$ коэффициент, не зависящий от температуры, $k_{\mathrm{B}}-$ постоянная Больцмана, $f-$ частота. Энергия 
активации $E_{a}$ соответствует разности положений потолка валентной зоны в кремнии $E_{V}^{\mathrm{Si}}$ и уровня Ферми $E_{\mathrm{F}}$ (рис. 2):

$$
E_{a}=E_{\mathrm{F}}-E_{V}^{\mathrm{Si}} .
$$

Измеряя температурную зависимость активной компоненты импеданса $G(T)$ барьера Шоттки со встроенной в область пространственного заряда квантовой ямой при различных частотах $f$, мы получаем семейство кривых с разным положением максимума проводимости. После этого строится зависимость $f / T_{\max }$ от обратной температуры в координатах Аррениуса. Аппроксимация данной зависимости линейной функцией дает энергию активации.

\section{3. Экспериментальные результаты}

На рис. 3 представлено семейство вольт-фарадных характеристик для образца, содержащего $6 \%$ олова. На низкочастотных зависимостях наблюдается плато в диапазоне напряжений $U=0.3-3.7 \mathrm{~B}$, обусловленное накоплением заряда в КЯ. Из вольт-фарадных характеристик был восстановлен профиль концентрации носителей заряда (рис. 4). На зависимости концентрации дырок от глубины наблюдается два пика. Основной пик, расположенный на глубине 0.2 мкм, мы ассоциируем с накоплением дырок в пленке $\mathrm{Ge}_{0.3} \mathrm{Si}_{0.64} \mathrm{Sn}_{0.06}$. Кроме основного пика в концентрации наблюдается дополнительный, который мы связываем с глубокими уровнями,

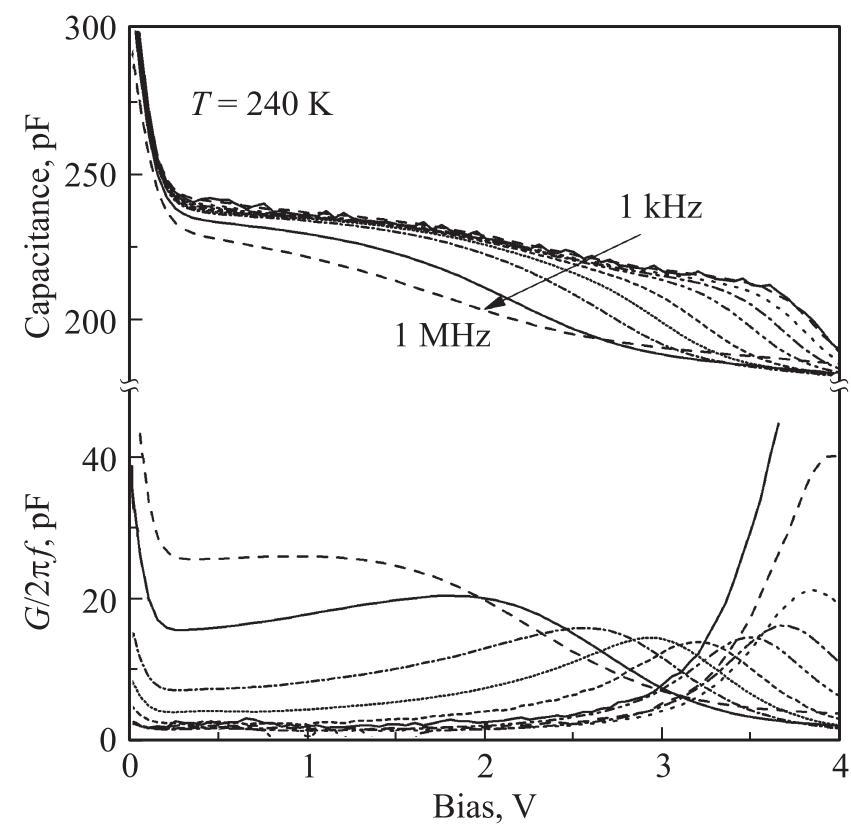

Рис. 3. Вольт-фарадные характеристики образца, содержащего твердый раствор $\mathrm{Si}_{0.64} \mathrm{Ge}_{0.3} \mathrm{Sn}_{0.06}$, при температуре $240 \mathrm{~K}$ и частотах, изменяющихся от 1 кГц до 1 МГц (вверху), и зависимости от приложенного смещения проводимости, нормированной на частоту $(G / 2 \pi f)$. По пику в низкочастотной проводимости определялось напряжение $U_{b}$, при котором носители заряда полностью уходят из ямы.

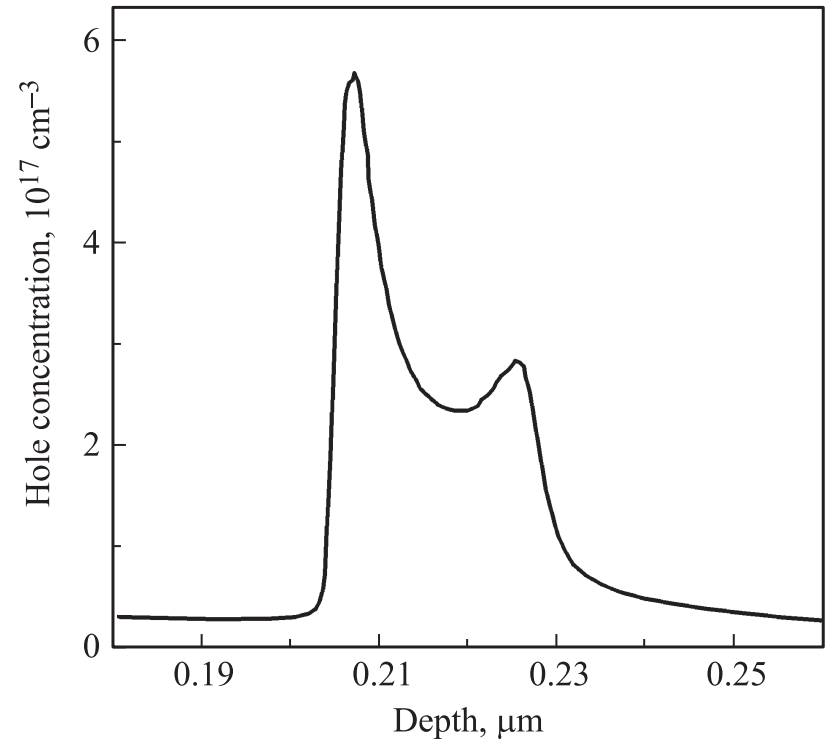

Рис. 4. Зависимость концентрации дырок от глубины, восстановленная из вольт-фарадной характеристики, в образце, содержащем $6 \%$ олова.

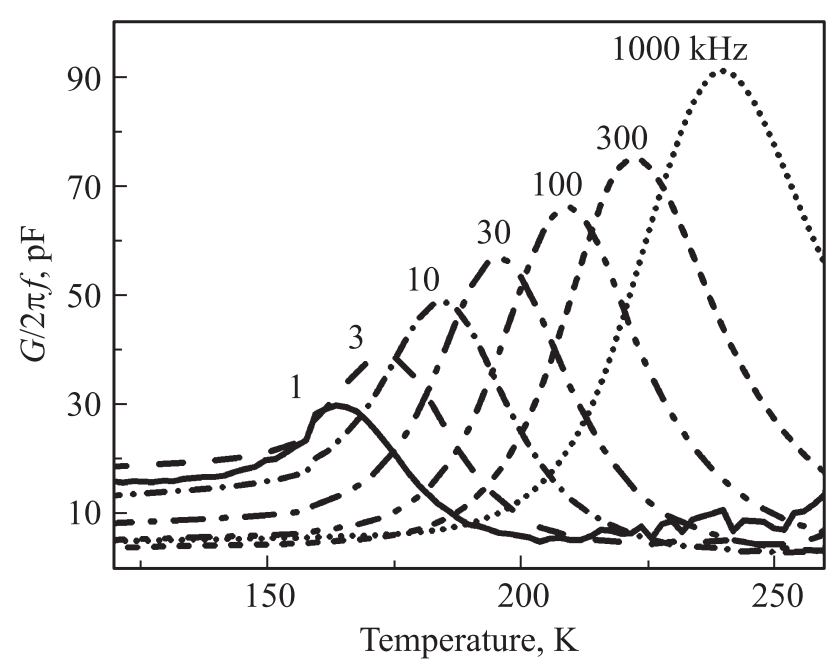

Рис. 5. Типичные зависимости проводимости от температуры при $U=0$ и различных частотах для образца с содержанием олова $6 \%$.

обусловленными структурными дефектами на гетерогранице пленка-〈окружающая кремниевая матрица $\rangle$. Интегрирование главного пика дает нам двумерную плотность дырок, находящихся в квантовой яме, которая составила величину $N_{s}=5 \cdot 10^{11} \mathrm{~cm}^{-2}$ для всех образцов.

На рис. 5 приведены температурные зависимости проводимости образца, содержащего твердый раствор $\mathrm{Ge}_{0.3} \mathrm{Si}_{0.64} \mathrm{Sn}_{0.06}$, снятые при различных частотах и нулевом постоянном смещении на образце. Амплитуда приложенного переменного сигнала составляла $50 \mathrm{MB}$. Из этих зависимостей определялась энергия активации темпа эмиссии дырок при заданном смещении с исполь- 
Энергия активации дырки и разрыв валентной зоны на гетерогранице $\mathrm{Si}_{2} \mathrm{Si}_{0.7-y} \mathrm{Ge}_{0.3} \mathrm{Sn}_{y}$ для напряженных тройных соединений при различном содержании олова, определенные экспериментально и по результатам математического моделирования

\begin{tabular}{|c|c|c|c|c|c|}
\hline $\begin{array}{c}\text { Содержание } \\
\text { олова }\end{array}$ & $E_{a}^{\exp }, э^{\mathrm{B}}$ & $E_{a}^{\text {calc }}, \ni \mathrm{B}$ & $\Delta E_{V}^{\text {calc }}, \ni \mathrm{B}$ & $\delta$, эВ & $\Delta E_{V}=E_{a}^{\exp }+\delta, \ni \mathrm{B}$ \\
\hline $4 \%$ & $0.24 \pm 0.01$ & 0.27 & 0.38 & 0.11 & $0.35 \pm 0.01$ \\
\hline $6 \%$ & $0.29 \pm 0.01$ & 0.36 & 0.48 & 0.12 & $0.41 \pm 0.01$ \\
\hline $10 \%$ & $0.43 \pm 0.01$ & 0.53 & 0.66 & 0.13 & $0.56 \pm 0.01$ \\
\hline
\end{tabular}

Примечание. $E_{a}^{\exp }$ - энергия связи дырки, полученная в эксперименте, $E_{a}^{\text {calc }}$ - энергия связи дырки, установленная в рамках 6-зонного $\mathbf{k p}$-моделирования, $\Delta E_{V}^{\text {calc }}-$ разрыв подзон тяжелых дырок валентной зоны, рассчитанный теоретически, $\delta$ - энергия размерного квантования, полученная из результатов моделирования как $\delta=\Delta E_{V}^{\text {calc }}-E_{a}^{\text {calc }}, \Delta E_{V}$ - разрыв подзоны тяжелых дырок валентной зоны, полученный из комбинации экспериментальных результатов и результатов математического моделирования. Значения $E_{a}^{\text {ехр }}$ получены усреднением величины энергии активации при различных смещениях, диапазоны смещений составляли: $0-0.52$ для образца, содержащего 4\% олова, $0-0.52$ для $6 \%$ олова и $0-0.208$ для $10 \%$ олова.

зованием выражений (1) и (2). Зависимость энергии активации от приложенного смещения приведена на рис. 6. Для корректного сравнения энергии активации в структурах с различным содержанием олова приложенное смещение нормировалось на напряжение, соответствующее полному опустошению квантовой ямы от дырок. На низкочастотной вольт-фарадной характеристике данное напряжение $U_{b}$ соответствует окончанию плато и пику в проводимости. Из приведенного рисунка видно, что энергия активации увеличивается с увеличением содержания олова в образце.

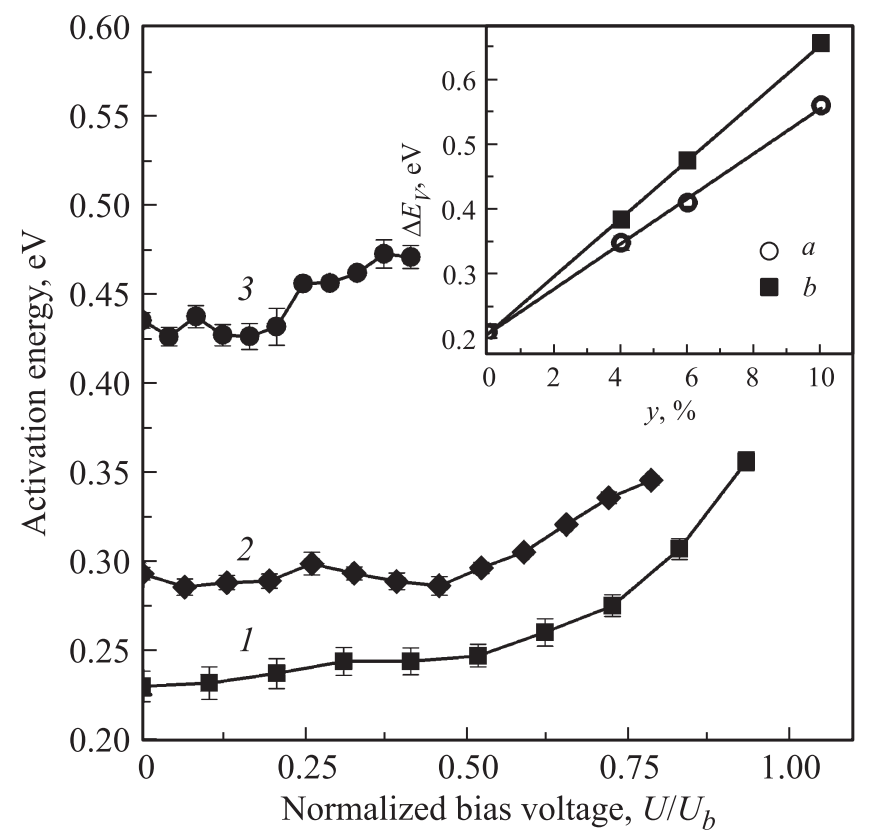

Рис. 6. Зависимости энергии активации дырки от приложенного смещения для образцов с различным содержанием олова: 1 - КЯ $\mathrm{Si}_{0.66} \mathrm{Ge}_{0.3} \mathrm{Sn}_{0.04}, 2$ - КЯ $\mathrm{Si}_{0.64} \mathrm{Ge}_{0.3} \mathrm{Sn}_{0.06}, 3$ - КЯ $\mathrm{Si}_{0.6} \mathrm{Ge}_{0.3} \mathrm{Sn}_{0.1}$. На вставке - зависимость разрыва валентной зоны от содержания олова: $a-$ экспериментальные значения, $b-$ результат, восстановленный из литературных данных; сплошные линии - аппроксимация зависимостей линейной функцией.
Разрыв валентной зоны может быть записан в виде следующего выражения (рис. 2):

$$
\Delta E_{V}=E_{a}+\left(E_{\mathrm{F}}-E_{0}\right)+\delta .
$$

Здесь $E_{0}$ - энергия основного состояния дырки в квантовой яме, $\delta$ - энергия размерного квантования. Для определения энергии Ферми использовалось простейшее соотношение для двумерных систем [27]:

$$
N_{s}=\frac{g_{v} m^{*}}{\pi \hbar^{2}}\left(E_{\mathrm{F}}-E_{0}\right)
$$

где $m^{*}$ - эффективная масса тяжелой дырки в квантовой яме, $g_{v}$ - кратность вырождения основного состояния дырки, $\hbar-$ постоянная Планка. Для концентраций дырок в КЯ $\sim 10^{12} \mathrm{~cm}^{-2}$, характерных для нашего случая, разность $E_{\mathrm{F}}-E_{0}$ имеет величину 5-6 мэВ, что меньше ошибки измерения энергии активации. Поэтому для дальнейшего определения разрыва валентной зоны мы полагали $E_{\mathrm{F}}-E_{0} \approx 0$. Так как КЯ в образцах, исследуемых в этой работе, имеет ширину $a=2 \mathrm{Hм}$, влияние размерного квантования на положение уровня дырки достаточно велико. Для получения информации о величине квантово-размерного эффекта были проведены численные расчеты спектра дырок в напряженной квантовой яме $\mathrm{Si}_{0.7-y} \mathrm{Ge}_{0.3} \mathrm{Sn}_{y} / \mathrm{Si}$.

\section{4. Моделирование}

Моделирование положения зон и спектра дырок в SiGeSn-гетероструктурах выполнялось в рамках 6-зонной kр-модели. Рассматривалась псевдоморфная пленка SiGeSn в матрице Si. Положение подзон валентной зоны в тройном растворе определялось по закону Вегарда в линейном по составу приближении [5]. Для учета поправок, связанных с деформациями пленки, постоянная решетки тройного соединения была восстановлена с учетом квадратичной нелинейности [5]. Деформации псевдоморфной пленки рассчитывались стандартным способом, описанным, например, в работе [28]. Важным фактором, влияющим на энергию связи дырки 
в квантовой яме, является значение латтинжеровских констант $\gamma_{1}, \gamma_{2}, \gamma_{3}$. Для твердых растворов $\mathrm{SiGe}$ известно, что зависимость параметров Латтинжера от состава нелинейна [29]. Для соединений GeSn подобных данных в литературе не имеется. Константы Латтинжера были определены с использованием следующей процедуры. Тройное соединение $\mathrm{SiGeSn}$ рассматривалось как двойное соединение $\left(\mathrm{Si}_{1-z} \mathrm{Ge}_{z}\right)_{y} \mathrm{Sn}_{1-y}$. Параметр твердого раствора $z$ выбирался таким образом, чтобы итоговое содержание $\mathrm{Ge}$ и $\mathrm{Si}$ имело заданное значение. После этого для соединения $\mathrm{Si}_{1-z} \mathrm{Ge}_{z}$ константы определялись по методике, предложенной в [29], а для тройного соединения использовалась линейная интерполяция между характеристиками $\mathrm{Si}_{1-z} \mathrm{Ge}_{z}$ и данными для олова, взятыми из [30]. Энергия размерного квантования дырки в КЯ была получена как разность положения потолка подзоны тяжелых дырок и энергии связи дырки в квантовой яме. Несмотря на то что энергия связи дырки $E_{a}^{\mathrm{calc}}$, рассчитанная в результате математического моделирования, существенно отличается от энергии связи дырки, измеренной экспериментально, энергия размерного квантования дырки слабо зависит от величины разрыва зон. Благодаря этому обстоятельству возможно определить „экспериментальный“ разрыв зон на гетерогранице $\mathrm{Si} / \mathrm{Si}_{0.7-y} \mathrm{Ge}_{0.3} \mathrm{Sn}_{y}$ как сумму энергии активации темпа эмиссии дырок, полученную из анализа аррениусовских зависимостей, и энергию размерного квантовая $\delta$, полученную из kр-моделирования. Для соединения $\mathrm{Si}_{0.7} \mathrm{Ge}_{0.3}$ величина разрыва валентной зоны взята из работы [31].

Полученные данные сведены в таблицу. Из таблицы видно, что при увеличении содержания олова в слое твердого раствора экспериментальная величина разрыва валентной зоны $\Delta E_{V}^{\exp }$ растет. Зависимость $\Delta E_{V}^{\exp }$ от состава может быть аппроксимирована линейной функцией $\Delta E_{V}^{\exp }=(0.21 \pm 0.01)+\left(3.35 \pm 7.8 \cdot 10^{-4}\right) y \quad$ (вставка к рис. 6). Рост разрыва валентной зоны между кремнием и слоем твердого раствора должен приводить к сдвигу края межзонного поглощения в область меньших энергий в гетероструктурах, содержащих квантовые ямы или сверхрешетки $\mathrm{Si} / \mathrm{SiGeSn} / \mathrm{Si}$. Именно такой характер зависимости края фундаментального поглощения от содержания олова наблюдался экспериментально в работе [13].

Еще одним фактом, который виден из таблицы, является систематическое завышение энергии связи дырки в квантовой яме, полученной из моделирования, по сравнению с экспериментом. Опираясь на известные из литературы значения разрыва валентных зон для гетеросистем $\mathrm{Si} / \mathrm{Ge}$ [31] и $\mathrm{Ge} / \mathrm{Sn}[5]$, можно восстановить теоретическое значение разрыва валентных зон в гетеросистеме $\mathrm{Si} / \mathrm{Si}_{0.7-y} \mathrm{Ge}_{0.3} \mathrm{Sn}_{y}$. Разрыв валентной зоны на гетерогранице, рассчитанный из литературных данных, аппроксимируется выражением $\Delta E_{V}^{\mathrm{calc}}=(0.21 \pm 0.0025)$ $+(4.45 \pm 0.05) y$. Большее значение энергии связи, полученной в рамках моделирования, может быть объяснено частичной релаксацией реальных пленок $\mathrm{SiGeSn}$ или неточным выбором латтинжеровских констант для слоя твердого раствора. Выяснение конкретной причины выходит за рамки данной статьи и является стимулом для дальнейших исследований.

\section{5. Заключение}

Методом спектроскопии адмиттанса измерены энергии связи дырок в гетероструктурах $\mathrm{Si} / \mathrm{Si}_{0.7-y} \mathrm{Ge}_{0.3} \mathrm{Sn}_{y} / \mathrm{Si}$ с квантовыми ямами различного состава. Установлено, что энергия связи дырки растет с увеличением содержания олова в квантовых ямах. Проведено сравнение экспериментальных результатов с результатами математического моделирования, полученными в рамках 6-зонного kp-метода. Установлено, что математическое моделирование дает завышенный результат по сравнению с экспериментом. Из результатов математического моделирования и экспериментальных результатов определена величина разрыва валентной зоны на гетероинтерфейсе $\mathrm{Si} / \mathrm{Si}_{0.7-y} \mathrm{Ge}_{0.3} \mathrm{Sn}_{y}$.

Исследования, описанные в работе, были поддержаны Российским фондом фундаментальных исследований (гранты № 16-32-60005-мол_а_дк, 16-32-00039-мол_а и 14-29-07153-офи_м). Также авторы хотят выразить благодарность В.В. Кириенко за помощь в приготовлении образцов и А.Г. Черкова за исследования структур с помощью просвечивающего электронного микроскопа.

\section{Список литературы}

[1] A. Elfving, A. Karim, G.V. Hansson, W.-X. Ni. Appl. Phys. Lett., 89, 083510 (2006).

[2] A.I. Yakimov, A.V. Dvurechenskii, Y.Y. Proskuryakov, A.I. Nikiforov, O.P. Pchelyakov, S.A. Teys, A.K. Gutakovskii. Appl. Phys. Lett., 75, 1413 (1999).

[3] A. Yakimov, V. Timofeev, A. Bloshkin, A. Nikiforov, A. Dvurechenskii. Nanoscale Res. Lett., 7, 494 (2012).

[4] N. Rappaport, E. Finkman, T. Brunhes, P. Boucaud, S. Sauvage, N. Yam, V. Le Thanh, D. Bouchier. Appl. Phys. Lett., 77, 3224 (2000).

[5] P. Moontragoon, R.A. Soref, Z. Ikonic. J. Appl. Phys., 112, 073106 (2012).

[6] A. Attiaoui, O. Moutanabbir. J. Appl. Phys., 116, 063712 (2014).

[7] R. Roucka, J. Mathews, R.T. Beeler, J. Tolle, J. Kouvetakis, J. Menéndez. Appl. Phys. Lett., 98, 061109 (2011).

[8] J. Mathews, R.T. Beeler, J. Tolle, C. Xu, R. Roucka, J. Kouvetakis, J. Menéndez. Appl. Phys. Lett., 97, 221912 (2010).

[9] M.-Y. Ryu, T.R. Harris, Y.K. Yeo, R.T. Beeler, J. Kouvetakis. Appl. Phys. Lett., 102, 171908 (2013).

[10] M. Oehme, D. Buca, K. Kostecki, S. Wirths, B. Holländer, E. Kasper, J. Schulze. J. Cryst. Growth, 384, 71 (2013).

[11] R.R. Lieten, J.W. Seo, S. Decoster, A. Vantomme, S. Peters, K.C. Bustillo, E.E. Haller, M. Menghini, J.P. Locquet. Appl. Phys. Lett., 102, 052106 (2013).

[12] H. Li, Y.X. Cui, K.Y. Wu, W.K. Tseng, H.H. Cheng, H. Chen. Appl. Phys. Lett., 102, 251907 (2013). 
[13] A.I. Nikiforov, V.A. Timofeev, A.R. Tuktamyshev, A.I. Yakimov, V.I. Mashanov, A.K. Gutakovskii. J. Cryst. Growth (2016). DOI: 10.1016/j.jcrysgro.2016.02.024.

[14] J.D. Gallagher, C.L. Senaratne, P. Sims, T. Aoki, J. Menéndez, J. Kouvetakis. Appl. Phys. Lett., 106, 091103 (2015).

[15] W. Du, Y. Zhou, S.A. Ghetmiri, A. Mosleh, B.R. Conley, A. Nazzal, R.A. Soref, G. Sun, J. Tolle, J. Margetis, H.A. Naseem, S.-Q. Yu. Appl. Phys. Lett., 104, 241110 (2014).

[16] W. Du, S.A. Ghetmiri, B.R. Conley, A. Mosleh, A. Nazzal, R.A. Soref, G. Sun, J. Tolle, J. Margetis, H.A. Naseem, S.Q. Yu. Appl. Phys. Lett., 105, 051104 (2014).

[17] V. D'Costa, C. Cook, A. Birdwell, C. Littler, M. Canonico, S. Zollner, J. Kouvetakis, J. Menéndez. Phys. Rev. B, 73, 125207 (2006).

[18] B.R. Conley, J. Margetis, W. Du, H. Tran, A. Mosleh, S.A. Ghetmiri, J. Tolle, G. Sun, R. Soref, B. Li, H.A. Naseem, S.-Q. Yu. Appl. Phys. Lett., 105, 221117 (2014).

[19] M.R. Bauer, C.S. Cook, P. Aella, J. Tolle, J. Kouvetakis, P.A. Crozier, A.V.G. Chizmeshya, D.J. Smith, S. Zollner. Appl. Phys. Lett., 83, 3489 (2003).

[20] M. Oehme, K. Kostecki, M. Schmid, M. Kaschel, M. Gollhofer, K. Ye, D. Widmann, R. Koerner, S. Bechler, E. Kasper, J. Schulze. Appl. Phys. Lett., 104, 161115 (2014).

[21] M. Jaros. Phys. Rev. B, 37, 7112 (1988).

[22] A.I. Nikiforov, V.A. Timofeev, S.A. Teys, A.K. Gutakovsky, O.P. Pchelyakov. Nanoscale Res. Lett., 7, 561 (2012).

[23] А.А. Блошкин, А.И. Якимов, В.А. Тимофеев, А.В. Двуреченский. ФТП, 48, 1065 (2014).

[24] А.И. Якимов, А.В. Двуреченский, А.И. Никифоров, Г.Ю. Михалев. Письма ЖЭТФ, 80, 367 (2004).

[25] A.I. Yakimov, A.I. Nikiforov, V.A. Timofeev, A.V. Dvurechenskii. Semicond. Sci. Technol., 26, 125002 (2011).

[26] K. Nauka, T.I. Kamins, J.E. Turner, C.A. King, J.L. Hoyt, J.F. Gibbons. Appl. Phys. Lett., 60, 195 (1992).

[27] T. Ando. Rev. Mod. Phys., 54, 437 (1982).

[28] J. Menéndez, J. Kouvetakis. Appl. Phys. Lett., 85, 1175 (2004).

[29] M. Rieger, P. Vogl. Phys. Rev. B, 48, 14276 (1993).

[30] T. Brudevoll, D.S. Citrin, M. Cardona, N.E. Christensen. Phys. Rev. B, 48, 8629 (1993).

[31] M. El Kurdi, S. Sauvage, G. Fishman, P. Boucaud. Phys. Rev. B, 73, 195327 (2006).

Редактор Л.В. Шаронова

\section{Valence-band offsets in strained SiGeSn/Si layers with different tin content}

A.A. Bloshkin 1,2, A.l. Yakimov ${ }^{1,3}$, V.A. Timofeev ${ }^{1}$, A.R. Tuktamyshev ${ }^{1}$, A.l. Nikiforov ${ }^{1,3}$, V.V. Murashov ${ }^{4}$

${ }^{1}$ A.V. Rzhanov Institute of Semiconductor Physics, Siberian Branch of Russian Academy of Sciences, 630090 Novosibirsk, Russia

2 Novosibirsk State University, 630090 Novosibirsk, Russia

3 Tomsk State University, 634050 Tomsk, Russia

${ }^{4}$ Novosibirsk Technical State University, 630073 Novosibirsk, Russia

Abstract Admittance spectroscopy was employed to study hole states in $\mathrm{Si}_{0.7-y} \mathrm{Ge}_{0.3} \mathrm{Sn}_{y} / \mathrm{Si}$ quantum wells with tin content $y=0.04-0.1$. It was found that the hole binding energy increases with increasing the tin content. Using the 6-band kp-method we determined the hole binding energy and valence band offsets in heterostructures $\mathrm{Si} / \mathrm{SiGeSn} / \mathrm{Si}$ with pseudomophic quantum well. Valence band offset at the $\mathrm{Si} / \mathrm{Si}_{0.7-y} \mathrm{Ge}_{0.3} \mathrm{Sn}_{y}$ heterointerface was obtained by combination of numerical calculation of hole confinement energy with the experimental determination of the hole binding energy. The valence band offset between pseudomorphic $\mathrm{Si}_{0.7-y} \mathrm{Ge}_{0.3} \mathrm{Sn}_{y}$ and $\mathrm{Si}$ obeys the dependence $\Delta E_{V}^{\exp }=(0.21 \pm 0.01)+\left(3.35 \pm 7.8 \cdot 10^{-4}\right) y \mathrm{eV}$. 